International Journal of Pure and Applied Mathematics

Volume 97 No. 2 2014, 231-240

ISSN: 1311-8080 (printed version); ISSN: 1314-3395 (on-line version)

url: http://www.ijpam.eu

doi: http://dx.doi.org/10.12732/ijpam.v97i2.12

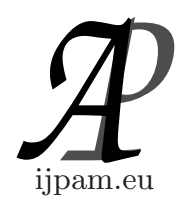

\title{
ON SOLVABILITY OF BOUNDARY VALUE PROBLEMS FOR SOLUTIONS OF THE STATIONARY SCHRÖDINGER EQUATION ON UNBOUNDED DOMAINS OF RIEMANNIAN MANIFOLDS
}

\author{
Elena Korolkova ${ }^{1}$, Sergei Korolkov ${ }^{2}$, Andrey Svetlov $^{3} \S$ \\ 1,2 Mathematics and IT Department \\ Volgograd State University \\ Pr. Universitetskiy, 100, Volgograd, 400062, RUSSIA
}

\begin{abstract}
In this paper we study the solutions of the stationary Schrödinger equation on unbounded domains of Riemannian manifolds with noncompact boundary. For the proper formulation of the boundary value problems we use an approach based on the introduction of equivalent functions class. We obtain sufficient conditions for solvability of some boundary value problems on such manifolds.
\end{abstract}

AMS Subject Classification: 58J32

Key Words: boundary value problem, $L$-harmonic function, Riemannian manifold, Dirichlet problem, model manifold

\section{Introduction}

The study of elliptic equations on Riemannian manifolds is up-to-date field of the contemporary mathematics and it's interface between differential geometry, mathematical analysis and theory of stochastic processes. An important class

Received: July 6, 2014

(C) 2014 Academic Publications, Ltd.

$\S$ Correspondence author url: www.acadpubl.eu 
of the problems in this field is Liouville-type theorems, which tell that spaces of bounded solutions of some elliptic equations on a manifold are trivial. Rather detailed description of the current state of research in this field can be found in $[2]$.

On the other hand, wide class of noncompact Riemannian manifolds admits the existence of nontrivial bounded solutions of elliptic differential equations. For example, it's true for simply connected Riemannian manifold with negative sectional curvature bounded away from zero and infinity as in [1] and [13]. Authors of the papers built a geometric compactification of manifold $M$ by adding a sphere $S(\infty)$ at the infinity and prove the solvability of the Dirichlet problem for $\bar{M}=M \cup S(\infty)$ on the construction of a harmonic function by a continuous boundary data on $S(\infty)$.

Note that the Dirichlet problem can be set on any non-compact Riemannian manifold if there is a natural compactification of it. In particular, it's possible for a spherically symmetric manifolds, model and quasimodel manifolds. Exact results on Liouville-type theorems and Dirichlet problem solvability for model and quasimodel manifolds were obtained in $[7,8,9,12]$.

However, on an arbitrary noncompact Riemannian manifold it can be difficult to set the Dirichlet problem. For this purpose a new approach to the setting of boundary value problems on noncompact Riemannian manifolds was proposed in [11]. It based on introducing the concept of equivalence class of functions and it allows to set the boundary value problems on manifolds with no natural geometric compactification (see also $[3,4,6,10]$ ).

All the above results refer to the case of the harmonic functions on noncompact Riemannian manifolds without a boundary (or with a compact boundary). The question is open for the case of manifolds with non-compact boundary: one can wonder how to set the boundary value problem in this case, what conditions are necessary and sufficient for the solvability of such problems.

We study the solutions of the stationary Schrödinger equation

$$
L u \equiv \Delta u-c(x) u=0,
$$

in the unbounded domains of the Riemannian manifolds with noncompact boundary. Here $c(x)$ is smooth non-negative function, and $c(x) \not \equiv 0$. From now on we call the solutions of (1) the $L$-harmonic functions. The aim of the paper is to obtain the conditions for solvability of boundary value problems for the $L$-harmonic functions in the domains under consideration.

Now we can give the exact statements. Let $M$ be connected noncompact smooth Riemannian manifold without boundary and $\Omega \subset M$ is simply connected unbounded domain with $C^{1}$-smooth boundary $\partial \Omega$. Let $\left\{B_{k}\right\}_{k=1}^{\infty}$ be a 
smooth exhaustion of $M$, that is the sequence of precompact open subsets of $M$ with $C^{1}$-smooth boundaries $\partial B_{k}$, and $M=\bigcup_{k=1}^{\infty} B_{k}, \bar{B}_{k} \subset B_{k+1}$ for all $k$. From now on we assume $B_{k} \cap \Omega \neq \emptyset, B_{k} \cap \Omega$ are simply connected, $\partial B_{k}$ and $\partial \Omega$ are transverse for all $k$. In this paper we have dealing with $L$-harmonic functions $u(x)$ on $M$ or on $\Omega$.

Let $f_{1}$ and $f_{2}$ are continuous functions on $M\left(\Omega\right.$ or $\partial \Omega$ ). We say $f_{1}$ and $f_{2}$ are equivalent on $M(\Omega$ or $\partial \Omega)$, and we write $f_{1} \stackrel{M}{\sim} f_{2}\left(f_{1} \stackrel{\Omega}{\sim} f_{2}\right.$ or $\left.f_{1} \stackrel{\partial \Omega}{\sim} f_{2}\right)$, if there is a smooth exhaustion $\left\{B_{k}\right\}_{k=1}^{\infty}$ of $M$ for which $\lim _{k \rightarrow \infty} \sup _{M \backslash B_{k}}\left|f_{1}-f_{2}\right|=0$ $\left(\lim _{k \rightarrow \infty} \sup _{\Omega \backslash B_{k}}\left|f_{1}-f_{2}\right|=0\right.$ or $\left.\lim _{k \rightarrow \infty} \sup _{\partial \Omega \backslash B_{k}}\left|f_{1}-f_{2}\right|=0\right)$. Relation " $\sim$ " is equivalence relation and it doesn't depend on exhaustion of $M([10,11])$.

Now we introduce a concept of $L$-potential of manifold $M$ with respect to a compact set $B \subset M$ (with $C^{1}$-boundary $\partial B$ ). We can assume without loss of generality that $B \subset B_{k}$ for all $k$. Let $\left\{v_{k}\right\}_{k=1}^{\infty}$ be a solution sequence of the following Dirichlet problems in $B_{k} \backslash B$

$$
\left\{\begin{array}{ccc}
L v_{k}=0 & \text { on } & B_{k} \backslash B \\
v_{k}=1, & \text { on } & \partial B \\
v_{k}=0 & \text { on } & \partial B_{k} .
\end{array}\right.
$$

By the maximum principle function sequence $\left\{v_{k}\right\}_{k=1}^{\infty}$ increase monotonically and has the limit $v_{M \backslash B}(x)=\lim _{k \rightarrow \infty} v_{k}(x)$, which is $L$-harmonic function on $M \backslash B$ and $0 \leq v_{M}(x) \leq 1$ on $M \backslash B$. Function $v_{M \backslash B}(x)$ is known as L-potential of manifold $M$ with respect to compact set $B$ (see also [11]).

The manifold $M$ is called $L$-strict if $L$-potential of manifold $M$ with respect to some compact set $B$ is equivalent to zero. This property does not depend on the choice of the compact set $B$ (see [11]).

Next we define $L$-potential of unbounded domain $\Omega$. Let's set $B_{k}^{\prime}=B_{k} \backslash \Omega$ and $v_{M \backslash B_{k}^{\prime}}$ is $L$-potential of manifold $M$ with respect to $B_{k}^{\prime}$. By the maximum principle the sequence $\left\{v_{M \backslash B_{k}^{\prime}}\right\}_{k=1}^{\infty}$ increase monotonically and it's bounded, so there is limit $v_{\Omega}$, which is $L$-harmonic in $\Omega, 0 \leq v_{\Omega} \leq 1$ and $\left.v_{\Omega}\right|_{\partial \Omega}=1$. Function $v_{\Omega}$ is called $L$-potential of $\Omega$.

Let $\Upsilon$ be connected unbounded subset of $M, v_{\Lambda}$ is $L$-potential of some set $\Lambda$, and $\Upsilon \backslash B \subseteq \Lambda$ for some compact set $B$. We assume $f_{1}$ and $f_{2}$ are continuous functions on $\bar{\Upsilon}$. We say that $f_{1}$ and $f_{2}$ are weakly equivalent on $\Upsilon \backslash B$ with respect to L-potential $v_{\Lambda}$, and we write $f_{1} \stackrel{\Lambda}{\simeq} f_{2}$ on $\Upsilon \backslash B$, if there is a constant $C$ for which

$$
\left|f_{1}-f_{2}\right| \leq C v_{\Lambda} \text { on } \overline{(\Upsilon \backslash B)} \text {. }
$$


Also we say that continuous function $f$ on $\Upsilon$ is in class of weakly admissible functions on $\Upsilon$ with respect to $L$-potential $v_{\Lambda}$, and we write $f \in K_{\Lambda}^{*}(\Upsilon)$, if for some compact set $B$ there is a $L$-harmonic function $u$ on $\Upsilon \backslash B$ for which

$$
u \stackrel{\Lambda}{\simeq} \text { on } \Upsilon \backslash B
$$

Remark 1. Let $\Upsilon \subseteq \Lambda, f_{1}$ and $f_{2}$ are continuous functions on $\bar{\Upsilon}$, and $B$ is a compact set. If $f_{1} \stackrel{\Lambda}{\simeq} f_{2}$ on $\Upsilon \backslash B$, then $f_{1} \stackrel{\Lambda}{\simeq} f_{2}$ on $\Upsilon$ by the maximum principle.

With regard to the Remark 1 from now on if $\Upsilon \subseteq \Lambda$ that the notation $f_{1} \stackrel{\Lambda}{\simeq} f_{2}$ means $f_{1} \stackrel{\Lambda}{\simeq} f_{2}$ on $\Upsilon$.

Remark 2. If $M$ is $L$-strict manifold and $B$ is a compact set, then $f \stackrel{M \backslash B}{\simeq} 0$ implies $f \stackrel{M}{\sim} 0$. The converse is not generally true (if $M$ is $L$-strict manifold, then $f \stackrel{M}{\sim} 0$ in general does not imply $f \stackrel{M \backslash B}{\simeq} 0$ for some compact set $B$ ).

Theorem 1. Let $B$ be a compact set and there is a $L$-harmonic function $u(x)$ on $\Omega \backslash B$. Then there are a constant $C$ and L-harmonic function $f$ on $\Omega$ for which

$$
|f-u| \leq C v_{M \backslash B} \text { on } \Omega \backslash B .
$$

The main result of the paper is the following theorem with a sufficient condition for solvability of our boundary value problem.

Theorem 2. Let $f \in K_{\Omega}^{*}(\Omega)$. If function $\varphi$ is continuous on $\partial \Omega$ and $\varphi \stackrel{\Omega}{\simeq} f$ on $\partial \Omega$, then there is a solution of the following problem on $\Omega$

$$
\left\{\begin{array}{c}
L u=0 \text { on } \Omega, \\
\left.u\right|_{\partial \Omega}=\varphi \\
u \stackrel{\Omega}{\simeq} f .
\end{array}\right.
$$

Remark 3. Theorems 1 and 2 generalize result of [10] for the case in which $M$ is $L$-strict and $\Omega=M$ (or $\Omega=M \backslash B$ for some compact set $B$ ). To be exact, in the case of $\Omega=M$ and $M$ is $L$-strict Theorem 1 with Remark 2 implies result of $[10]$ (Theorem 1 , implication $(i i i) \Rightarrow(i)$ ). In the case when $M$ is $L$-strict and $\Omega=M \backslash B$, where $B$ is a compact set, implication $(i i i) \Rightarrow(i i)$ of Theorem 1 from the cited paper follows from the Theorem 2. Backward implications are obvious. 
Note that we assume $c(x) \not \equiv 0$. In the case of $c(x) \equiv 0$ a stationary Schrödinger equation becomes a Laplace-Beltrami equation. $L$-harmonic functions are different from harmonic functions (that is the solutions of the LaplaceBeltrami equation). For instance, on a manifold without boundary the space of bounded harmonic functions is trivial if and only if the space of nonnegative harmonic functions is trivial (e.g. [2]). It's not true for $L$-harmonic functions (e.g. [6]). Results on solvability of some boundary value problems for harmonic functions in unbounded domains of Riemannian manifolds and in cones of model manifolds can be found in [5].

\section{Proof of the Theorem 1}

Lemma 1. If $B$ is a compact set, then $f_{1} \stackrel{\Omega}{\simeq} f_{2}$ on $\Omega \backslash B$ if and only if $f_{1} \stackrel{\Omega \backslash B}{\simeq} f_{2}$ on $\Omega \backslash B$

Proof. Assume that $f_{1} \stackrel{\Omega \backslash B}{\simeq} f_{2}$ on $\Omega \backslash B$. By the definition of weak equivalence we have $\left|f_{1}-f_{2}\right| \leq C v_{\Omega \backslash B}$ with some constant $C$.

By the maximum principle $0<d=\inf _{\partial B \cap \Omega} v_{\Omega}<1$. Comparison principle for $\frac{v_{\Omega}}{d}$ and $v_{\Omega \backslash B}$ in $\Omega \backslash B$ implies $\frac{v_{\Omega}}{d} \geq v_{\Omega \backslash B}$. It leads to $f_{1} \stackrel{\Omega}{\simeq} f_{2}$ on $\Omega \backslash B$.

The proof in the opposite direction follows from the maximum principle.

Lemma 2. Let $B$ be a compact set. Then $v_{M \backslash B} \stackrel{\Omega}{\simeq} 0$ on $\Omega \backslash B$.

Proof. Assume that $\Omega \cap B=\emptyset$.

Let's again $\left\{B_{k}\right\}_{k=1}^{\infty}$ be a smooth exhaustion of $M$ and $B_{k}^{\prime}=B_{k} \backslash \Omega$. Then $B \subset B_{k}^{\prime}$ starting from some $k$ and by the maximum principle $v_{M \backslash B}<v_{M \backslash B_{k}^{\prime}}$ on $M \backslash B_{k}^{\prime}$ starting from some $k$. Passing to the limit here as $k \rightarrow \infty$ we have $v_{M \backslash B} \leq v_{\Omega}$ on $\Omega$, which implies the required result.

Next we assume $\Omega \cap B \neq \emptyset$. Then $v_{M \backslash B} \stackrel{\Omega \backslash B}{\simeq} 0$ on $\Omega \backslash B$, as proved above. Now we apply Lemma 1 and obtain the required result.

Proof of the Theorem 1. Let's $u$ be continuously extended by zero on $\Omega \cap B$.

Assume that $\left\{B_{k}\right\}_{k=1}^{\infty}$ is a smooth exhaustion of $M$ and $\Omega \cap B_{k} \neq \emptyset, \partial \Omega$ and $\partial B_{k}$ are transversal for all $k$, and $B \subset B_{k}$ for all $k$.

We set $\Omega(k)=\partial\left(B_{k} \cap \Omega\right), \Omega(0)=\partial B \cap \Omega$. 
Consider the sequence of functions $\left\{\varphi_{k}\right\}_{k=1}^{\infty}$ that are solutions to the problem

$$
\left\{\begin{array}{l}
L \varphi_{k}=0 \text { on } B_{k} \cap \Omega, \\
\left.\varphi_{k}\right|_{\Omega(k)}=\left.u\right|_{\Omega(k)},
\end{array}\right.
$$

First we prove that $\varphi_{k}$ is uniformly bounded on $\Omega(0)$. Assume the contrary. Then there is a subsequence $\left\{k_{n}\right\}$ for which $a_{k_{n}}=\max _{\Omega(0)}\left|\varphi_{k_{n}}\right| \rightarrow \infty$ as $n \rightarrow \infty$. We set $k_{n}=k$ and $\Phi_{k}=\varphi_{k} / a_{k}$ on $B_{k} \cap \Omega$. Then

$$
\left\{\begin{array}{ccc}
\Phi_{k}=u / a_{k} & \text { on } & \partial\left(B_{k} \cap \Omega\right) \backslash B \\
\Phi_{k}=0 & \text { on } & \partial \Omega \cap B, \\
\max _{\Omega(0)}\left|\Phi_{k}\right|=1 . & &
\end{array}\right.
$$

By the maximum principle for function $\Phi_{k}-\frac{u}{a_{k}}$ on $\left(B_{k} \cap \Omega\right) \backslash B$ and next on $B \cap \Omega$, we have

$$
-1-\frac{\max _{\Omega(0)}|u|}{a_{k}}+\frac{u}{a_{k}} \leq \Phi_{k} \leq 1+\frac{\max _{\Omega(0)}|u|}{a_{k}}+\frac{u}{a_{k}} \quad B_{k} \cap \Omega .
$$

Actually, $\max _{\Omega(0)}\left|\Phi_{k}\right|=1$ implies that

$$
-1 \leq \Phi_{k} \leq 1 \text { on } \Omega(0)
$$

It leads to

$$
-1-\frac{\max _{\Omega(0)}|u|}{a_{k}} \leq \Phi_{k}-\frac{u}{a_{k}} \leq 1+\frac{\max _{\Omega(0)}|u|}{a_{k}} \Omega(0) .
$$

From this and

$$
\Phi_{k}-\frac{u}{a_{k}}=\frac{\varphi_{k}}{a_{k}}-\frac{u}{a_{k}}=\frac{u}{a_{k}}-\frac{u}{a_{k}}=0 \text { on }(\partial \Omega \backslash B) \cup\left(\partial B_{k} \cap \Omega\right)
$$

it follows that

$$
-1-\frac{\max _{\Omega(0)}|u|}{a_{k}} \leq \Phi_{k}-\frac{u}{a_{k}} \leq 1+\frac{\max _{\Omega(0)}|u|}{a_{k}} \text { on }\left(B_{k} \cap \Omega\right) \backslash B .
$$

On the other hand, from the maximum principle it follows that

$$
-1 \leq \Phi_{k} \leq 1 \text { on } B \cap \Omega
$$

because $\Phi_{k}=0$ on $\partial \Omega \cap B$ and $\max _{\Omega(0)}\left|\Phi_{k}\right|=1$. 
Combining the estimates (4) and (5), we obtain (3).

From (3) it follows that $\left\{\Phi_{k}\right\}_{k=1}^{\infty}$ is locally uniformly bounded on $\Omega$. It implies the existence of a subsequence of $\left\{\Phi_{k}\right\}$ that is uniformly convergent to a limit function $\Phi$ on any compact subset of $\Omega$ and $L \Phi=0$ on $\Omega,\left.\Phi\right|_{\partial \Omega \cap B}=0$ and $-1 \leq \Phi \leq 1$ on $M$. Next, we can take a suitable subsequence of $\left\{\Phi_{k}\right\}$ such that $\max _{\Omega(0)}|\Phi|=1$. Thus, we reach a contradiction with a maximum principle.

Therefore, the assumption $a_{k_{n}}=\max _{\Omega(0)}\left|\varphi_{k_{n}}\right| \rightarrow \infty$ as $n \rightarrow \infty$ is wrong, and it implies that the sequence $\varphi_{k}$ is uniformly bounded on $\Omega(0)$. It follows that the sequence $\left\{\varphi_{k}-u\right\}_{k=1}^{\infty}$ is locally uniformly bounded on $\Omega$ and then it leads to existence of $f=\lim _{k \rightarrow \infty} \varphi_{k}, L f=0$.

As we showed above $\bar{a}=\sup _{k} \max _{\Omega(0)}\left|\varphi_{k}\right|<\infty$. The maximum principle and $\left.\varphi_{k}\right|_{\Omega(R)}=\left.u\right|_{\Omega(K)}$ imply

$$
u-\left(\bar{a}+\max _{\Omega(0)} u\right) v_{M \backslash B} \leq \varphi_{k} \leq u+\left(\bar{a}+\max _{\Omega(0)} u\right) v_{M \backslash B}
$$

on $\left(\Omega \cap B_{k}\right) \backslash B$.

Passing here to the limit as $k \rightarrow \infty$ we obtain the required result.

So the proof of Theorem 1 is completed.

\section{Proof of the Theorem 2}

Lemma 3. Let $B$ be a compact set and $g$ is a continuous $L$-harmonic function on $M \backslash B$. Then for each continuous function $\Phi$ on $\partial B$ there is a solution of the following problem on $M \backslash B$

$$
\left\{\begin{array}{c}
L u=0 \text { on } M \backslash B, \\
\left.u\right|_{\partial B}=\Phi, \\
M \stackrel{M}{\simeq} g .
\end{array}\right.
$$

Proof. Theorem 1 implies that there are a constant $C$ and $L$-harmonic function $w$ on $M$ such that

$$
|g-w| \leq C v_{M \backslash B} \text { on } M \backslash B .
$$

It follows that $w^{M \backslash B} \stackrel{\simeq}{\simeq}$ on $M \backslash B$.

Just like in [10] (proof of implication $(i) \rightarrow(i i)$ of Theorem 1) we prove the existence of $L$-harmonic function $u$ on $M \backslash B$ such that $\left.u\right|_{\partial B}=\Phi$,

$$
|u-w| \leq\left(\max _{\partial B}|\Phi|+1\right) v_{M \backslash B},
$$


and it implies the required result.

If we set $g \equiv 0$ in Lemma 3 condition and $w \equiv 0$ in its proof, we come to the following assertion.

Corollary 3. Let $B$ be a compact set. Then for each continuous function $\Phi$ on $\partial B$ there is a solution of the following problem on $M \backslash B$

$$
\left\{\begin{array}{c}
L u=0 \text { on } M \backslash B, \\
\left.u\right|_{\partial B}=\Phi, \\
M \backslash B \\
u \stackrel{ }{\simeq} 0,
\end{array}\right.
$$

such that $|u| \leq\left(1+\max _{\partial B}|\Phi|\right) v_{M \backslash B}$.

Proof of the Theorem 2. We start with the proof of the Theorem 2 in the case of $f \equiv 0$. It means that for any continuous function $\varphi$ on $\partial \Omega$ such that $\varphi \stackrel{\Omega}{\simeq} 0$ on $\partial \Omega$ there is a $L$-harmonic function $w$ on $\Omega$ for which $\left.w\right|_{\partial \Omega}=\varphi$ and $w \stackrel{\Omega}{\simeq} 0$.

We denote by $\hat{f}$ a continuous bounded extension of $\varphi$ from $\partial \Omega$ to $M$. Let's again $\left\{B_{k}\right\}_{k=1}^{\infty}$ be a smooth exhaustion of $M, B_{k}^{\prime}=B_{k} \backslash \Omega$.

Corollary 3 implies the existence of the following problem's solution in $M \backslash$ $B_{k}^{\prime}$

$$
\left\{\begin{array}{c}
L w_{k}=0 \text { on } M \backslash B_{k}^{\prime}, \\
\left.w_{k}\right|_{\partial B_{k}^{\prime}}=\left.\hat{f}\right|_{\partial B_{k}^{\prime}}, \\
M \backslash B_{k}^{\prime} 0,
\end{array}\right.
$$

such that

$$
\left|w_{k}\right| \leq\left(1+\left.\max _{\partial B_{k}^{\prime}} \hat{f}\right|_{\partial B_{k}^{\prime}}\right) v_{M \backslash B_{k}^{\prime}}
$$

It follows from (6) that the family of functions $\left\{w_{k}\right\}_{k=1}^{\infty}$ on $\Omega$ is uniformly bounded because $\hat{f}$ is bounded. Let $w(x)$ be a limit function. We remark that $L w=0,\left.w\right|_{\partial \Omega}=\varphi$ since $\left.w_{k}\right|_{\partial B_{k}^{\prime}}=\left.\hat{f}\right|_{\partial B_{k}^{\prime}}$ and $\left.\hat{f}\right|_{\partial \Omega}=\varphi$. Moreover, passing in (6) to the limit as $k \rightarrow \infty$, we obtain

$$
|w| \leq\left(1+\sup _{M} \hat{f}\right) v_{\Omega}
$$

hence $w \stackrel{\Omega}{\simeq} 0$.

Next we consider $f \in K_{\Omega}^{*}(\Omega) \not \equiv 0$. By the definition of class $K_{\Omega}^{*}(\Omega)$ it implies that there are a compact set $B$ and a $L$-harmonic function $g$ on $\Omega \backslash B$ for which

$$
|g-f| \leq C v_{\Omega} \text { on } \Omega \backslash B
$$


Theorem 1 implies that there are a constant $C_{1}$ and $L$-harmonic function $v$ on $\Omega$ such that

$$
|g-v| \leq C_{1} v_{M \backslash B} \text { on } \Omega \backslash B .
$$

It follows from (7), (8) and Lemma 2 that

$$
v \stackrel{\Omega}{\simeq} f \text { on } \Omega \backslash B
$$

The Remark 1 and $\varphi \stackrel{\Omega}{\simeq} f$ on $\partial \Omega$ lead to

$$
v \stackrel{\Omega}{\simeq} f
$$

and

$$
v \stackrel{\Omega}{\simeq} \varphi \text { on } \partial \Omega .
$$

As follows from the above, there is a solution of the following problem on $\Omega$ :

$$
\left\{\begin{array}{c}
L w=0 \text { on } \Omega, \\
\left.w\right|_{\partial \Omega}=\varphi-\left.v\right|_{\partial \Omega}, \\
w \stackrel{\Omega}{\simeq} 0 .
\end{array}\right.
$$

It's obvious that $u \equiv w+v$ is the required function. The proof of Theorem 2 is completed.

\section{Acknowledgments}

The reported study was partially supported by RFBR, research project No. 13-01-97038-r_povolzhye_a.

\section{References}

[1] M.T. Anderson, The Dirichlet problem at infinity for manifolds with negative curvature, J. Diff. Geom., 18, no. 4 (1983) 701-721.

[2] A. Grigor'yan, Analitic and geometric background of recurrence and nonexplosion of the Brownian motion on Riemannian manifolds, Bull. Amer. Math. Soc., 36, no. 2 (1999), 135-249. DOI: 10.1090/S0273-0979-9900776-4 
[3] S.A. Korol'kov, Harmonic functions on Riemannian manifolds with ends, Siberian Math. J., 49, no. 6 (2008), 1051-1061. DOI: 10.1007/s11202-0080101-1

[4] S.A. Korolkov, A.G. Losev, Solutions of elliptic partial differential equations on Riemannian manifolds with ends, Vestnik VolGU [in Russian], 1 (14) (2011), 23-40.

[5] E.A. Korolkova, S.A. Korolkov, Boundary problems for harmonic functions on unbounded open sets of Riemannian manifolds, Vestnik VolGU [in Russian], 1 (18) (2013), 45-58.

[6] S.A. Korolkov, A.G. Losev, Generalized harmonic functions of Riemannian manifolds with ends, Mathematische zeitschrift, 272, no. 1-2 (2012), 459472. DOI: 10.1007/s00209-011-0943-2

[7] A.G. Losev, Some Liouville theorems on Riemannian manifolds of a special type, Soviet Math. (Iz. VUZ), 35, no. 12 (1991), 1523.

[8] A.G. Losev, On a criterion for the hyperbolicity of noncompact Riemannian manifolds of a special form, Math. Notes, 59, no. 3-4 (1996), 400404. DOI: $10.1007 / \mathrm{BF} 02308689$

[9] A.G. Losev, E.A. Mazepa, Bounded solutions of the Schrödinger equation on Riemannian products, St. Petersburg Math. J., 13, No. 1 (2001), 57-73.

[10] A.G. Losev, E.A. Mazepa, V.Y. Chebanenko, Unbounded solutions of the Stationary Shrödinger equation on Riemannian manifolds, Computational Methods and Function Theory, 3: 2 (2003), 443-451. DOI: 10.1007/BF03321048

[11] E.A. Mazepa, Boundary value problems for the stationary Shrödinger equation on Riemannian manifolds, Siberian Math. J., 43, no. 3 (2002), 473-479. DOI: 10.1023/A:1015411502059

[12] M. Murata, Positive harmonic functions on rotationary symmetric Riemannian manifolds, Potential Theory (1992), 251-259. DOI: $10.1515 / 9783110859065.251$

[13] D. Sullivan, The Dirichlet problem at infinity for a negatively curved manifolds, J. Diff. Geom, 18: 4 (1983), 723-732. 